\title{
Collagenase and sodium iodoacetate-induced experimental osteoarthritis model in Sprague Dawley rats.
}

\begin{abstract}
The objective of this study was to apply and compare two different experimental osteoarthritis (OA) methods in the rat, namely: Collagenase induced OA (CO) and Monosodium iodoacetate induced OA (MIA) models. The assessment of OA development and progression were performed through three different periods (2, 4 and 6 weeks). Intraarticular injection of either $4 \mathrm{mg}$ joint-1 CO type II or $3 \mathrm{mg}$ joint-1 MIA, were administered to the adult male Sprague Dawley rats, into their right knee joints. Evaluation of OA changes in the knees was achieved with both histopathology score system and radiography approach. Gross results revealed earliest changes such as swelling and redness of the right knee joints of all rats injected with either CO or MIA. Joint dissection revealed distinct thickening of the joint capsule in MIA-injected rats than in $\mathrm{CO}$ group. Present finding revealed early development of radiographical as well as histopathological changes in MIA injected group. However, both OA injected groups resulted in a chronic joint degeneration, measured by cellular changes, matrix degradation, subchondral changes and marginal osteophyte formation. Present findings showed significantly higher histopathological score in MIA injected group than those of $\mathrm{CO}$ in each of the three selected periods for OA induction. In conclusion, present results demonstrated that MIA can induce OA changes in a shorter period of time than CO in the Sprague Dawley rat. Radiography approach could be a useful tool to evaluate osteoarthritic changes in the knee joints.
\end{abstract}

Keyword: Osteoarthritis; Cartilage; Rat; MIA; Collagenase; Histopathology. 